\title{
The Ontological Content of Pre-predicative Experience
}

\author{
Yajun $\mathrm{Wu}^{\mathrm{a}}{ }^{\mathrm{*}}$, Xia Kang ${ }^{\mathrm{b}}$ \\ a School of Foreign Languages and Literature, Yunnan Normal University, Kunming-650500, China. \\ ${ }^{b}$ Faculty of Education, The University of Hong Kong, Hong Kong-999077, China. \\ *Corresponding author Email: wuyajun1225@163.com \\ DOI: https://doi.org/10.54392/ajir2149 \\ Received: 09-11-2021; Revised: 24-12-2021; Accepted: 25-12-2021; Published: 27-12-2021
}

Abstract: From epistemological and methodological perspectives, the pre-predicative experience is essential for the self-performed knowledge assessment. Husserl speaks about the "self-evident" knowledge in the context of phenomenological reduction, and the concept of self-evidence is postulated to unfold as part of pre-predicative experience. However, Husserl does not explain the content of the latter. This article conducts a comprehensive study of the attributes of pre-predicative experience and precedent phenomenon alongside three dimensions of order, category, and function. The analysis argues that the pre-predicative experience is influenced by precedent knowledge.

Keywords: Ontological Content, Phenomenology, Pre-Predicative Experience, Precedent Phenomenon, SelfEvidence of Knowledge

\section{Introduction}

As a category, Husserl's pre-predicative experience provokes discussions with a breakdown into reflectionbased descriptive analysis, which in turn constitutes a normal practice in the theory of knowledge (Farber, 1940; Pradelle, 2020). However, the ontological content of pre-predicative experience is implicit. Traditionally, logic refers to the categorical judgment that can be reduced to a form "All S are p" (Husserl 1974). This mainly applies to the nature of logical products, regardless of the legitimizing source of formal logic (Kant, 2004). That is to say, philosophers blurred the line between " $p$ " and higher-order subjects. Given this, the traditional logicians seldom concern with whether the constitutions of judgment have a self-sufficient knowledge basis or not (DeLay, 2019; Staiti, 2015). Many philosophers such as Descartes, Hume, and Kant have widely discussed the foundation for testifying the self-evidence of knowledge but it was not articulated until Husserl, who grounded the concept in the frame of pre-predicative experience (Staiti, 2018). Descartes takes the phrase "I think, therefore I am" as the first principle to argue that "I" is a distinct subject to start from in the journey towards self-evidence. According to Descartes, "ego" serves a reliable basis for the verification of empirical knowledge, while Husserl criticizes this statement, proclaiming "ego" to exist in a spiritual, rather than substantial, form. The substantialization of "ego" is part of transcendental realism that is somewhat absurd (Yu, 2010), as this practice inevitably leads to solipsism. Hume also denies the substantialization of "ego". In view of Hume, sensuous perceptions congenitally lay ground for the establishment of genuine knowledge (Giorgi, 2014; Hume, 2009). In other words, Hume emphasizes the association of empirical intuition and inner perception and believes that one's sensuous perception lays the foundation for elucidation of the self-sufficiency of knowledge. "The Hume problem" transforms into a problem of inductive method reliability (Lange, 2008). With or without intention, he blazed a trail for Kant and his transcendental philosophy. Hume's philosophy is based on traditional psychology and thus fails to explore the transcendental subject enclosed in the argument for the self- sufficiency of knowledge. Taking "the Hume problem" as a starting point, Kant investigates the self-sufficiency of knowledge through the lens of transcendentality. He is also the first philosopher to coin the concept of transcendental subject and give insights into phenomenology correctly (Husserl, 1983; Rouba, 2020). However, the emergence of phenomenology in Kantianism is a forced compromise (Yu, 2003). There are still some remnants of empirical psychology in Kant's argument for the self-sufficiency of knowledge. While Hume makes 
a point of subject's empirical intuition, Kant expresses a philosophical vision that is not transcendentally pure. Although Kantianism contributed the notion of transcendental subject, it was not further developed. Hence, the relevant, up-to-date concept of transcendental subject cannot be articulated. Husserl extends the system of knowledge about judgments and considers both predicate experience and pre-predicative experience (Zhang, 2004). The content of pre-predicative experience constitutes the general knowledge, which helps interpret one's own intuition and experience by means of phenomenological reduction. In terms of function, pre-predicative experience provides a set of self-sufficient evidence so that predicate experience can be obtained. Husserl was the first to associate the self-evidence of knowledge with pre-predicative experience.

To demonstrate the self-evidence of knowledge, Husserl takes "predicate judgment" and "pre-predicative experience" as the core concepts and develops the theory of transcendental logic. According to Husserl, predicative experience reaches genealogical lucidity when backtracking to the pre-predicative experience. I doing so, the object is "revealed" and the truthiness of predicate experience is proven. Unlike Descartes, the Husserl's genealogical investigation of the predicate experience addresses the original and untheorized opinions. In other words, Husserl's argument for the self-evidence of knowledge is the most substantial as compared to others (Audi, 2019; Bostar, 1987). However, Husserl carries out his philosophical reflections by following the Cartesian principle of "I think, therefore I am." In this frame, the "subject" will eventually be materialized. In the Husserl's philosophical system, the concept of "subject" will thus transcend other objects that are outside the concept so that the Husserl's thought would inevitably fall into solipsism. Heidegger argues that the "subject" in Husserlian philosophy embraces the idea of an object, rather than an object itself (Wu, 2017). Post-phenomenology also disagrees with the Husserl's assertion, holding that the subject is not substantive but co-exists with other subjects. In the argument for self-evident knowledge, Husserl sketches the idea of pure transcendental logic to eliminate drawbacks of dogmatic absolutism and skeptical relativism (Bostar, 1987). His argument is of considerable relevance to epistemology and methodology. However, the underlying entity of all phenomena was not revealed due to the lack of a categorical apparatus permitting the articulation of the Husserl's pre-predicative experience. To close this gap, the paper aims to explore and articulate the ontological content of pre-predicative experience that can prove the self-evidence of knowledge by resorting to the precedent phenomenon.

\section{Methods}

This study utilized the following methods:

- deductive means, when exploring attributive characteristics;

- synthesis of research experience, when identifying similarities between a precedent phenomenon and predictive experience;

- extrapolation method, to draw illustrative examples;

- hierarchical organization of formal and substantive abstractions, when identifying the nature of prepredicative experience according to Husserl's theory.

\section{Results}

Husserl introduced the term "pre-predicative experience" to prove its positive relation to the phenomenological reduction of predicate experience. To demonstrate whether the precedent phenomena can serve as the ontological content of the pre-predictive experience, it is necessary to explore the attributes of these two notions alongside three dimensions of sequence, category, and function.

\section{The dimension of logical sequence}

Pre-predicative experience is a crucial concept in the Husserl's theory of phenomenological reduction. According to Husserl, the self-evidence of knowledge can be expounded within the context of subject's prepredicative experience (Reeder, 1984). The philosopher had two reasons to distinguish the pre-predicative experience. 
First, Husserl denied both the existence of absolute truth (Bostar, 1987) and the sufficiency of sensuous perceptions (Follesdal, 2010). In the view of Husserl, the "truth" objectively does not exist and is relational at the core. That is to say, the nature of truth changes over time and can be reflected retrospectively under the guidance of teleology. Regards the sensuous perception, there is that one famous example in the Husserlian philosophy, the seeing of a dice, which demonstrates that an individual can only perceive a specific aspect or part of the object being observe at the certain point in time (Follesdal, 2006). Hence, the individual's perception of an object is always incomplete due to the infinite number of combinations of noemata and cannot constitute a foundation for the verification of empirical knowledge. Meanwhile, Husserl also denies the existence of a predicate in Platonic sense. The inadequacy of sensuous perception and disregard for the predicate mean that arguments for the self-evidence of knowledge according to Descartes and Hume are condemned to failure. For these reasons, Husserl drag the said argument into the light of pre-predicative experience. The Husserl's views of necessary truth and perceptual sufficiency are strikingly parallel to the Wittgenstein's conviction. Wittgenstein criticizes the Moore's common sense theory, postulating that an individual's perception is not trustworthy. Wittgenstein also holds a relativist view of truth, arguing that the certainty of propositions is born out from the logical priorities of propositions the subject has, rather than his cognitive perception of them (Willams, 2004).

Second, Husserl had a rather specific conception of philosophy and contributed to the evolution of transcendental phenomenology in the field of self-evidence of knowledge. Husserl set his mind on pursuing the rigorous scientific nature of philosophy instead of the "peculiarities of special forms" (Husserl, 1965). Therefore, the Husserl's philosophical investigation aims to establish a universal theory, rather than specific philosophical problems. In Husserl's view, only the pure consciousness of a perceptual object can be regarded as the strict and undoubted grasp of this object. To ensure the strict scientificity of philosophy, Husserl develops a transcendental phenomenological path so that the self-evidence of knowledge can be achieved through continuous retrospection.

To sum up, Wittgenstein states that any content that lays foundation for the logical evaluation of knowledge has a priority over the content that lacks this potential, while Husserl points at the psychological factor of selfevidence. It is said that the self-evidence of knowledge can be grasped by intuition. In other words, the subject's intuitive data are regarded as "the final test of truth" (Spiegelberg, 1942) in which the pre-predicative experience resembles an all-sufficient background for the validation of predicate experience $(\mathrm{Ni}, 2003)$. In short, the prepredicative experience is a priori logical ( $\mathrm{Ni}, 2014)$. To put this simply, the pre-predicative experience logically precedes the subject's predicate experience.

\section{The dimension of category}

Pre-predicative experience is external to and beyond the subject's consciousness. Husserl denies the possibility that an individual's perception can lay the foundation for predicate experience. In Husserl's words, "only one aspect of the object comes within the purview of this realization in imagination, although more and more aspects can be brought therein" (Husserl, 1990). That is, the subject's perception of an object is not and never will be complete due to the diversity of subjective observation perspectives. Similarly, Qian (2015) argues that "a concept is continuously enriched or modified under the combined effect of multiple predictions" (Qian, 2015). Hence, an individual's knowledge of the perceived object is always incomplete and thus cannot lay the foundation for the demonstration of self-evident knowledge. Take a "horse" as an example, an individual's perception of a horse improves and changes in concert with her or his cognitive perspectives:

- A horse runs fast;

- A horse runs slow;

- Horses are herbivores;

- Horses can live for up to 50 years;

- Horses were an excellent means of transportation in ancient times.

The examples above indicate that an individual's perception of a horse is not permanent, and it is almost impossible to identify a horse, as its aspects imaginatively vary. A subject's knowledge of the perceived object will increasingly become abundant but a complete understanding of the perceived object's content is not to be reached 
(Livingston, 2018). Subjects may have diverse and sometimes even contradictory intuitive perceptions of the same object depending on the context of perception. For instance, the subject might think, "horses run fast," comparing to walking. If the parallels were made with vehicles such as airplanes and high-speed trains, the subject would feel that "horses run slow." However, these two contradictory propositions are not necessarily in conflict with that how a subject perceives the object. The incompleteness of perception and the compatibility of contradictory views both indicate that the individual experience cannot serve as the logical starting point in the argument for self-evident knowledge. According to Husserl, the pre-predicative experience logically precedes the predicate judgment, which constitutes the precondition of predicative proposition. The cognitive abilities of the subject and the functional setting of pre-predicative experience imply that it is impossible to reasonably demonstrate the necessity for knowledge in person perception. Overall, the pre-predicative experience does not lean on the individual's cognitive domain but is external to it.

Husserl's argumentation of inter-subjectivity also testifies that the pre-predicative experience has a certain objectivity value. Since 1905, Husserl has turned to the theme of inter-subjectivity, focusing on the problem of other minds. The investigation he went for was aimed to answer if it is possible for multiple minds to share the same prepredicative experience. The possibility of reaching a consistent understanding of anything through communication has been disclaimed, for people are not mind readers and can only communicate via mediating structures (Enfield, 2000). This is why the pre-predicative experience exists outside the cognitive domain. The Putnam's argument is more direct in claiming that a logical starting point for perception is outside one's mind and cannot be regarded as such for the formation of belief (Putnam, 2016).

\section{The dimension of function}

In view of Husserl, only a direct experience of the object has the absolute truth and can be regarded as givenness (Miron, 2016). As argued by Husserl, the subject can intuitively comprehend the essence of the object by winnowing out the irrelevant judgments (Wang, 2003). Therefore, Husserl investigated the self-evidence of knowledge not through the lens of predicate experience but retrospectively by addressing the part of knowledge that cannot be expressed in words (Zhang, 2004), the pre-predicative experience. For the phenomenological reduction of predicate experience, the pre-predicative experience functions as norms, which govern empirical science (Livingston, 2018). Thus, it plays an essential role in the demonstration of both the self-evident nature of formal logic and interactive subjectivity. In short, it is inevitably related to the manifestation of self-evidence of knowledge and enables the mutual understanding between individuals.

Based on the above analysis, there are three attributes of pre-predicative experience: (1) logically, comes before predicative judgment; (2) is external to the individual's cognitive domain; (3) functionally, contains sufficient information to ensure the truth of predicate experience. However, Husserl does not make an inquiry into the ontological content of the pre-predicative experience, for two reasons at least. On the one hand, he strictly abided by the principle of transcendental philosophy, meaning he was only interested in the original, purely conscious intuitiveness of experience $(\mathrm{Ni}, 2014)$. On the other hand, the lack of ontological framework is closely related to the Husserl's pursuit of philosophical elitism (Zhou, 2008). His interest in philosophical investigation was rather theoretical (Zhou, 2008), meaning that he was likely to use deductive methods and verify empirical experience by employing the pre-predicative experience. Although Husserl expounds much on the signification and function of the prepredicative experience, the lack of an epistemological framework signifies that the foundation for the self-evidence of knowledge remains unclear. Thus, the Husserl's argument for the self-evidence of knowledge is incomplete and constitutes some unknown components.

With respect to attributes from the above, a comparative analysis of pre-predicative experience and precedent phenomenon was conducted. The analysis shows that these two concepts share the same attributes. Both are of greater importance that subjectivity, both transcend the necessity of knowledge, and both are external to one's mind. Thus, both become the objective reality. In terms of content, the pre-predicative experience is neither congenital nor empty but originates in the acquaintance and transmission of precedent knowledge. That is, the precedent phenomenon acts on the cognitive psychology of individuals and encourages them to interpret their predicate experience. Like the pre-predicative experience, the precedent phenomenon can lay the foundation for the self-evidence of empirical knowledge. 
The empirical knowledge refers to the unity of form and content. Regards the content, its self-evidence cannot be justified simply by deducing from empirical data. The way in which the cause-and-effect relationship between pre-predicative experience and empirical knowledge was found is also important. In other words, how can the pre-predicative experience of content ground the self-evidence of predicate proposition?

\section{Discussion}

The precedent phenomenon is referred to as a component of the ontological content of pre-predicative experience. To reveal similarities between precedent phenomenon and pre-predictive experience, this section explores attributes of the former.

\section{Precedent phenomenon}

A cultural group is the most basic social unit, which is mainly shaped by specific precedent phenomena. According to Goddard, a "homogeneous group" means a group where members mutually share similar precedent phenomena (Goddard, 2005). This embraces two different aspects. First, the spectrum of precedent phenomena defines what features will distinguish cultural groups and for this, people from the same cultural group share similar lifestyle, thinking, and behavior. Second, group members of the same kind communicate with each other (Goddard, 2005). Hence, other individuals within the group influence individual behavior or value orientations of a single member. At this point, behavioral patterns and value orientations promoted within the group have a significant effect on how an individual behaves and judges the world. Not only behavioral patterns and value orientations of one person evolve in response to the external knowledge but they also serve a standard for other group members. In short, individuals belong to a specific cultural group and communicate with each other through the precedent phenomena.

The precedent phenomenon refers to psychological facts about the social and cultural life of people (Wang, 2011), which play a central role in shaping the psychology of a cultural group. Within a cultural community, individuals may have countless experiences. However, not all psychological facts can acquire the status of a precedent phenomenon. Only those facts that are known to the most members of the group can add to its cognitive foundation and become precedent phenomena, which shape the attributes of culture.

There are four units of precedent phenomena (Milostivaya, 2016): precedent texts, precedent names, precedent statements, and precedent situations. Together with a sketchy representation of ethnic identity, they form a trinity of cultural markers (Figure 1). Specifically, the precedent phenomena consist of signifiers (precedent names and precedent statements), signified (precedent texts and precedent situations), and a concept which the first two unit categories denote (Kashima et al., 2013).

The minimal representation of ethnic stipulation (Concept)

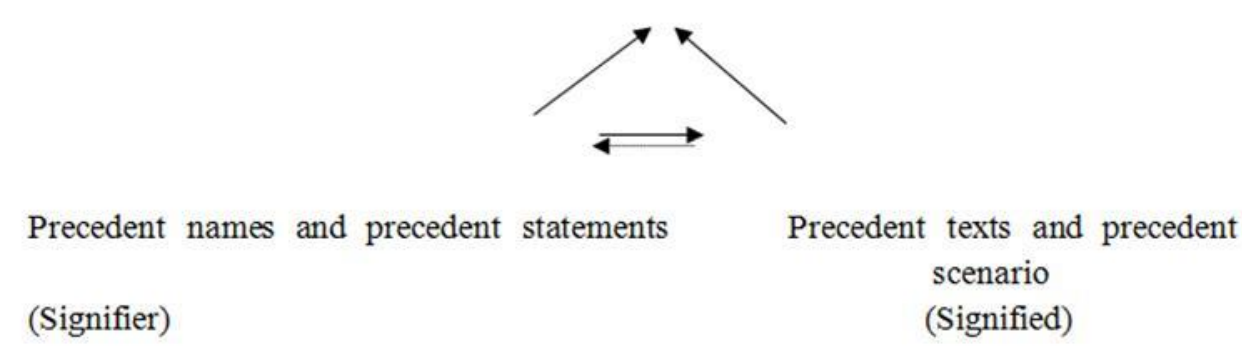

Figure 1. Components of the precedent phenomenon

The absorption of diverse precedent units is closely linked to the cultural group. First, it does not occur overnight. Each precedent phenomenon goes through stages of selecting, processing, shaping, and collective identification of a cultural group. Second, the precedent phenomenon is not permanent. As the context of social life transforms, some precedent phenomena no longer adapt to the cognitive foundation of the group. Other, however, better reflect and describe the new context and thus enter the mentality of members of a cultural group. In short, an individual absorbs the precedent phenomena of a specific cultural group in which he or she turns out to be. 
Attributes of the precedent phenomenon

Precedent phenomena are inherited in cultural groups. Diverse texts or situations are labeled as precedent if known to the most members of the cultural group. In terms of function, the precedent phenomenon serves as the fundamental part of cultural group cognition and the mutually shared information is believed to be central to communication and mutual understanding between individuals.

First, members of a cultural group grasp the knowledge, beliefs, and assumptions that are specific to their cultural group by absorbing precedent phenomena (Clark, 1996). In doing so, they socialize. This means that logically, the precedent phenomena have precedence over the individual existence, which is also consistent with the Wittgenstein's vision.

Second, the precedent phenomenon embodies the unique way of life, thinking, and behavior (Goddard, 2005). It is carried within the cultural group through intergenerational transmission. In terms of category, the precedent phenomenon transcends the subjectivity of ego and manifests the objective reality. In other words, the precedent phenomenon is external to the individual's mind. This conclusion is consistent with Putnam's thought.

Many scholars have investigated the effect of precedent phenomena on mutual comprehension (Kronmuller \& Barr, 2015). According to Hall, the precedent phenomena mediate the communication of minds. Thus, the meaning of mental representations is understood via mediating structures (no-telepathy assumption) (Hutchins \& Hazlehurst, 1995). Hall (1959) argues that one's reflection on the external world is inevitably patterned by culture (Hall, 1959). Further, he points out that cultural patterns serve a means of information exchange that helps individuals understand each other (Hall, 1959). To sum up, individuals shearing the same culture can reach mutual understanding through a common mediation structure, the precedent phenomena.

\section{Conclusion}

Husserl was aimed at explaining the foundation of empirical knowledge within the context of pre-predicative experience and transcendental logic. However, he did not discuss the ontological content of pre-predicative experience, focusing on the internal time-consciousness. Therefore, this paper focused on the ontological content of pre-predicative experience and explored the original content of knowledge that can lay foundation for the selfevidence of empirical knowledge. Firstly, the attributes of pre-predicative experience and precedent phenomenon were discussed alongside three dimensions of sequence, category, and function. Secondly, both concepts were analyzed to find shared attributes. Finally, the ontological content of pre-predicative experience was discussed based on the analysis results. The article concludes that the ontological content of pre-predicative experience is neither congenital nor empty nor arbitrary but is influenced by precedent phenomena.

\section{References}

Audi, R., (2019), Understanding, self-evidence, and justification, Philosophy and Phenomenological Research, 99(2), 358-381. https://doi.org/10.1111/phpr.12492

Bostar, L.J., (1987), The methodological significance of Husserl's concept of evidence and its relation to the idea of reason, Husserl Studies, 4, 143-167. https://doi.org/10.1007/BF00365250

Clark Herbert, H., (1996), Communities, commonalities, and communication. In S. C. Levinson \& J. Gumperz (Eds.), Rethinking Linguistic Relativity Cambridge: Cambridge University Press, 324-355.

DeLay, S., (2019), Some remarks on phenomenology's past, Metascience, 28, 335-340. https://doi.org/10.1007/s11016-019-00395-0

Enfield, N.J., (2000), The theory of cultural logic: How individuals combine social intelligence with semiotics to create and maintain cultural meaning, Cultural Dynamics, 12(1), 35-64. https://doi.org/10.1177\%2F092137400001200102

Farber, M., (1940), Edmund Husserl and the Background of his Philosophy, Philosophy and Phenomenological Research, 1(1), 1-20. https://doi.org/10.2307/2103193

Follesdal, D., (2006). Husserl's reductions and the role they play in his phenomenology In H. L. Dreyfus \& M. A. Wrathall (Eds.), A companion to phenomenology and existentialism Blackwell Publisher, 105-113. 
Follesdal, D., (2010), Ultimate justification in Husserl and Wittgenstein, Philosophical Analysis, 1(2), 45-59.

Giorgi, A., (2014), Phenomenological philosophy as the basis for a human scientific psychology, Humanistic Psychologist, 42(3), 233-248. https://doi.apa.org/doi/10.1080/08873267.2014.933052

Goddard, C., (2005), The lexical semantics of culture, Language Sciences, 27(1), 51-73. https://doi.org/10.1016/j.langsci.2004.05.001

Hall, E.T., (1959), The silent language, Doubleday \& Company, New York.

Hume, D., (2009), A treatise of human nature, The Floating Press, Aukland.

Husserl, E., (1965), Phenomenology and the crisis of philosophy, Harper \& Row Publishers, New York.

Husserl, E., (1983), General introduction to a pure phenomenology, Martinus Nijhoff Publishers. Boston.

Husserl, E., (1990). The idea of phenomenology, Kluwer Academic Publishers, Dordrecht.

Hutchins, E., \& Hazlehurst, B., (1995), How to invent a shared lexicon: The emergence of shared form-meaning mappings in interaction, Cambridge University Press, 53-67. https://doi.org/10.1017/CB09780511621710.005

Kant, I., (2004), Critique of pure reason, People's Publishing House, Beijing.

Kashima, Y., Lyons, A., \& Clark, A., (2013), The maintenance of cultural stereotypes in the conversational retelling of narratives, Asian Journal of Social Psychology, 16(1), 60-70. https://doi.org/10.1111/ajsp.12004

Kronmuller, E., \& Barr, J.D., (2015), Referential precedents in spoken language comprehension: A review and metaanalysis, Journal of Memory and Language, 83, 1-19. https://doi.org/10.1016/j.jml.2015.03.008

Lange, M., (2008), Hume and the problem of induction, Handbook of the history of logic, 10, 43-91. https://doi.org/10.1016/B978-0-444-52936-7.50002-1

Livingston, P.M., (2018), Edmund Husserl: From intentionality to transcendental phenomenology, Philosophy of mind in the nineteenth century, Routledge, 232-248. https://doi.org/10.4324/9780429508134-14

Milostivaya, A., (2016), Precedent phenomena in M. Bulgakov's works as reflected in their English and German translations, Rupkatha Journal on Interdisciplinary Studies in Humanities, 8(1), 113-121.

Miron, R., (2016), Husserl and other phenomenologists, European Legacy, 21(5-6), 467-480. https://doi.org/10.1080/10848770.2016.1181353

$\mathrm{Ni}$, L., (2003), The notion of pure logics in Husserl's logical investigation, Jianghai Academic Journal, 5, 29-35.

$\mathrm{Ni}$, L., (2014), Husserl's phenomenology of interactive subjectivity, Journal of Sun Yet-Sen University (Social Science Edition), 54(3), 83-91.

Pradelle, D., (2020), On Husserl's concept of the pre-predicative: Genealogy of logic and regressive method, Language and phenomenologyhenomenology, Routledge, New York.

Putnam, H., (2016), Naturalism, realism, and normativity, Harvard University Press, Massachusetts

Qian, G., (2015), "Horse" gives no concept of a horse-philosophic significance of predicate and predication, Foreign Language Research, 186(5), 1-4.

Reeder, H.P., (1984), A phenomenological account of the linguistic mediation of the public and the private, Husserl Studies, 1, 263-280. https://doi.org/10.1007/BF01569218

Rouba, M.V., (2020), The paradox of Kant's transcendental subject in German philosophy in the late eighteenth century, Kantian Collection, 39(2), 7-25. https://doi.org/10.5922/0207-6918-2020-2-1

Spiegelberg, H., (1942), Phenomenology of direct evidence, Philosophy and Phenomenological Research, 2(4), 427456. https://doi.org/10.2307/2103084

Staiti, A., (2015), Husserl and Rickert on the nature of judgment, Philosophy Compass, 10(12), 815-827. https://doi.org/10.1111/phc3.12270

Staiti, A., (2018), Pre-predicative experience and life-world: Two distinct projects in Husserl's late phenomenology, The Oxford handbook of the history of phenomenology, Oxford University Press, 155-172. DOI: 10.1093/oxfordhb/9780198755340.013.12 
Wang, H., (2003), Sensation and being within the horizons of intuition of essence-An analysis of Husserl's thinking of intuition, Journal of Nanjing University, 40(4), 22-29.

Wang, Z., (2011), An exploration of the precedent phenomena in language, Heilongjiang University Press, Harbin. Willams, M., (2004), Wittgenstein, truth and certainty, Wittgenstein's lasting significance, Routledge, London.

Wu, Z., (2017), The problem of origin in Husserlian phenomenology, World Philosophy, 3, 20-28.

Yu, H. (2003), Kant's approaching to phenomenology-from critique of pure reason to critique of judgment, Jiangsu Social Sciences, 3, 35-39.

Yu, H., (2010), From "cogito, ergo sum" to "Ich bin, ego cogito", Hubei Social Sciences, 12, 102-105.

Zhang, T., (2004), An analysis of Husserl's theory of pre-predictive experience, Philosophical Research, 3, 29-38.

Zhou, X., (2008), Husserl's theory on the philosophical ideal and the crisis of European philosophy, Journal of Graduate School of Chinese Academy of Social Sciences, 6, 39-45.

Funding: No funding was received for conducting this study.

Conflict of Interest: The Authors have no conflicts of interest to declare that they are relevant to the content of this article.

\section{About The License}

(C) The Author(s) 2021. The text of this article is open access and licensed under a Creative Commons Attribution 4.0 International License

\section{Cite this Article}

Yajun Wu \& Xia Kang, The Ontological Content of Pre-predicative Experience, Asian Journal of Interdisciplinary Research, 4(4) (2021) 80-87. https://doi.org/10.54392/ajir2149 